\title{
Yawning: A Possible Confounding Variable in EMG Biofeedback Studies
}

Biofeedback and Self-Regulation, Vol. 14, No. 4, 1989

\author{
R. E. Oman \\ Centre de Recherche, Institut de Réadaptation de Montieal, and Université de Montieal \\ S. J. Sullivan \\ Centre de Recherche, Institut de Réadaptation de Montieal, and Concordia University \\ J. Fleury
}

Service de Physiatrie, Institut de Réadaptation de Montréal

E. Dutil

Centre de Recherche, Insitut de Réadaptation de Montréal, and Université de Montréal

Centre de Recherche, Institut de Réadaptation de Montréal, 6300 Avenue Darlington, Montréal, Quebec, Canada H3S

The electrical activity recorded from a resting or contracting muscle represents the output of the lower motoneuron pool or final common pathway innervating that muscle. As such, it is reflective of a wide range of central neurophysiological processes, both facilitatory and inhibitory. Thus, the observed EMG signal may reflect unrelated muscle activity in addition to the electrical activity owing to the variable under investigation. Such extraneous information may include crosstalk (Wolf, 1978), unrelated voluntary contractions (Delwaide \& Toulouse, 1981), and changes in posture (Hayes \& Sullivan, 1976). Although professionals are aware of the peripheral spread of EMG signals as a potential confounding variable, they are not necessarily aware of the central, neural, and reflexive augmentation that can occur. This paper will demonstrate the extent of this augmentation and that it can occur inadvertently with a commonly occurring event, yawning.

A recent report by Wimalaratna and Capiledo (1988) described a case of an acute hemiplegic patient who was unable to voluntarily contract the muscles of the arm (a manual muscle test score of 0 on a 5-point scale) yet was observed to move the arm reflexively while yawning. This observation is in keeping with that described by Mulley (1982), who found that 31 of the 40 hemiplegic patients he questioned reported that their plegic arm moved involuntarily during yawning. The physiology of yawning and its relation to respiration are not well documented (Wimalaratna \& Capiledo, 1988; Forrester, 1988), but the observation of increased muscle activity in the paretic or plegic limb suggests that it may have a general facilitatory effect on central nervous system activity, although the exact mechanisrn is yet to be determined.

A similar pattern of increased muscle activity during yawning in a hemiplegic patient was observed in our laboratory during an EMG biofeedback training program. We were fortuitous in being able to document EMG levels in the anterior deltoid muscle both during yawning and during a series of attempted maximum voluntary contractions. The purpose of this paper is to present these data and to discuss the possible consequences of yawning in the interpretation of data obtained in EMG biofeedback studies. 


\section{METHOD}

Subject

The subject was a 71-year (right hand dominant) male with left-sided hemiplegia as a consequence of a cerebrovascular accident 6 months previously. The patient was not experiencing any other health-related problems and was not taking any medication that would influence the outcome of the study. At the time of the data collection he was undergoing a comprehensive rehabilitation program and, in addition, was participating in an optional EMG biofeedback training program.

Prior to beginning the biofeedback training program, his stage of motor recovery was assessed as being at level 1 (the lowest possible score) on the Brunnstrom scale (Brunnstrom, 1956) and a score of 4 on a possible of 66 on the Fugl-Meyer test (Fugl-Meyer, Jaasko, Leyman, Olsson, \& Steglind, 1975). This score reflects the subject's inability to dissociate synergistic movements during an attempted voluntary contraction of the upper limb. The patient was unable to perform isolated movements of the upper extremity (e.g., elbow flexion) without contracting a number of other muscle-related groups (e.g., shoulder flexors). Despite the obvious plegic nature of the patient's upper extremity (he was virtually unable to move his arm) as evidenced by these scores, he was highly motivated and interested in the training program. He was, however, continually fatigued and occasionally yawned. While he was yawning, his "plegic" arm was often observed to move with components of both shoulder and elbow flexion.

\section{Procedure}

The details of the testing procedures were presented elsewhere (Sullivan, Oman, Mathieu, Arsenault, \& Dutil, 1988) and are presented in summary form here. Before, immediately after, and 1 month following an EMG biofeedback training program, the electrical activity of the anterior deltoid was recorded together with the motor and functional performance of the hemiplegic/paretic limb. During each of the 12 training sessions the patient was seated in a chair that provided maximum support and isolated the performance of the shoulder flexor muscle group. He was required to produce a maximum voluntary isometric contraction of the shoulder flexors of the noninvolved limb followed by the hemiplegic limb. The electrical activity of the contracting anterior deltoid was recorded (surface electrodes placed 3 centimeters apart) during each 5-second contraction. The patient was instructed to produce a maximum voluntary contraction and to match both the level and pattern of the electrical activity (linear envelope) produced by the hemiplegic limb to that previously generated by the noninvolved limb for each trial. The subject looked straight ahead at the computer screen on which the resulting feedback signals were presented. Ten trials were recorded for each training session.

The recorded EMG signals were amplified (CMRR > $100 \mathrm{~dB})$ and filtered $(10-500 \mathrm{~Hz})$ prior to being sampled $(1,000 \mathrm{~Hz})$ and digitized by an IBM PC/AT, which generated the "real-time" EMG displays that the subject viewed. The sampled EMG data were also trans ferred to a PDP 11 $123+$ for subsequent off-line analysis. The 5 seconds of data underwent a series of mathematical signal processing stages as follows. The signal was full wave rectified and filtered at $6 \mathrm{~Hz}$, and the peak (and integrated) EMG values were obtained for each trial.

During four different training sessions EMG data were obtained during at least part of a yawn. These data were obtained while the patient was resting between trials. Since yawning is an involuntary action, and therefore not predictable, data acquisition began when the researcher observed the "start" of a yawn. Consequently, data corresponding to the beginning of the yawn were not collected. The computer program was designed to collect 5 seconds of data from the point 
of initiation. The data were not obtained in conjunction with an attempted voluntary contraction. Synergistic contractions of the hemiplegic limb were observed during yawning. Owing to the restrained nature of the subject's trunk and arm position, the most obvious movement was that of elbow flexion.

Table I. Peak EMG (Microvolts) During Maximum Voluntary Isometric

\begin{tabular}{|c|c|c|c|}
\hline & \multicolumn{2}{|c|}{ Maximum contraction $^{a}$} & \multirow{2}{*}{$\frac{\text { Yawning }}{\text { Hemiplegic }}$} \\
\hline & Noninvolved & Hemiplegic & \\
\hline $\begin{array}{l}\text { Trial 1 } \\
\text { (Session 2) }\end{array}$ & $85.8 \pm 9.81$ & $4.33 \pm 0.52$ & 85.0 \\
\hline $\begin{array}{l}\text { Trial } 2 \\
\text { (Session 6) }\end{array}$ & $125.90 \pm 19.70$ & $11.50 \pm 1.69$ & 118.0 \\
\hline $\begin{array}{l}\text { Trial } 3 \\
\text { (Session 10) }\end{array}$ & $141.4 \pm 17.08$ & $4.80 \pm 1.12$ & 23.0 \\
\hline $\begin{array}{l}\text { Trial } 4 \\
\text { (Session 12) }\end{array}$ & $151.67 \pm 15.87$ & $10.11 \pm 3.87$ & 90.0 \\
\hline
\end{tabular}

\section{RESULTS}

The peak values obtained from the rectified and filtered EMG signal recorded from the plegic limb for four 5-second intervals of yawning are presented in Table 1. Also shown are the means and standard deviations of peak EMG for both limbs for the 10 trials recorded during the same training session. For each training session there is a marked reduction in the peak EMG recorded from the plegic limb in comparison with that recorded from the noninvolved limb during an attempted maximum contraction. For each of the four episodes of yawning the peak EMG is markedly increased, compared with the session means and standard deviations for the limb. This increase is clearly beyond the normal variation of the hemiplegic limb, and in some instances (Trials 1 and 2) the peak EMG values for the hemiplegic limb fall within the range of values recorded from the noninvolved limb for that particular session.

Sample data are shown in Figure 1. Here it can be seen that the peak EMG value is a realistic representation of the recorded signal, which is indeed elevated for a large part of the signal. Measures of the integrated EMG were not used in this analysis owing to their lack of comparative meaning. It should be noted that it was impossible to record the signal in its entirety because of the involuntary nature of yawning. 

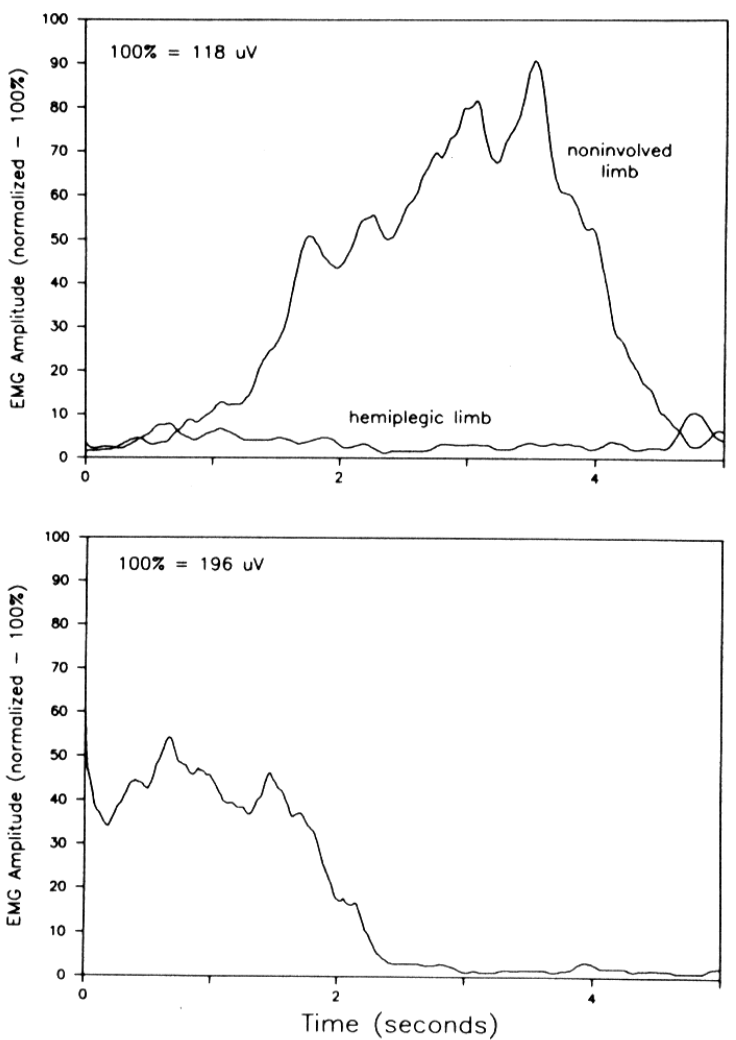

Fig. 1. The pattern of EMG activity (linear envelope) recorded for a single trial during a sequential maximum isometric shoulder flexion by the noninvolved and hemiplegic arms (upper figure) and the EMG recorded from the hemiplegic arm during yawning (lower figure). For both figures the data have been normalized in such a manner that the peak amplitude of the contraction of the noninvolved limb served as a reference for determining the appropriate axis for the the subsequent contraction by the hemiplegic limb. The $100 \%$ value (microvolts) is shown for each figure. For the single yawning trial, the data are expressed as a function of the preceding trial (not shown). The subject had commenced yawning when the data collection began.

\section{DISCUSSION}

Yawning, an involuntary motor response most probabIy triggered from brainstem centers (Wimalaratna \& Capiledo, 1988), appears to be capable of providing a central drive or facilitation to peripheral motoneurons in the hemiplegic patient. We documented a marked increase in only a single muscle; however, coupled with our clinical observations of the development of a general synergistic flexion of the plegic limb, this clearly suggests a central facilitation of the otherwise flaccid limb.

The changes observed here support previous observations (Mulley, 1982; Wimalaratna \& Capiledo, 1988) and also document the magnitude of the facilitation at the level of the individual muscle. The facilitation allowed the muscle to obtain an output level approximate to that recorded from the homologous muscle of the noninvolved side. This occurred for two of the four episodes of yawning. The other two episodes also resulted in greatly increased electrical output. This facilitation may be likened to the changes observed with the use of facilitatory/inhibitory techniques by physical therapists in the treatment of CNS disorders (Bobath, 1978). The use of such techniques requires the patient to either voluntarily or passively adopt a specified posture in order 
to provide the desired facilitation/inhibition. Obviously, an involuntary phenomenon such as yawning cannot be used in the same manner. The observations in this case clearly suggest that the peripheral motoneurons are capable of being activated if the appropriate central drive is provided.

In EMG biofeedback studies, changes in the level of electrical activity recorded from the contracting muscle are used both for feedback purposes and for the direct quantification of the muscle activity. Fundamental to the use of the EMG signal is the fidelity of the signal at both the technical (Cram \& Garber, 1986; Sullivan, Mathieu, \& Goulet, 1989) and physiological levels (Basmajian, 1985). The observations reported here clearly suggest that the

EMG signal could be contaminated by yawning, thus leading to possible misinterpretation of a particular value. A related concern is the possibility of greatly increased variability over a series of trials. Just one episode of yawning would inflate the variability of a training session, leading to difficulty in interpreting the data. The data presented in Table 1 clearly show the degree of variability associated with the recording of peak EMG values in a controlled laboratory situation. Peak EMG recordings that represent the single greatest value recorded during the contraction are inherently variable both within and between recording sessions. This, coupled with the changes due to training (e.g., trials 1 to 4 represent a difference of 12 training sessions) may account for changes in both absolute values and the associated variability observed in the present data. In a clinical situation, there may very well be a further increase in variability, and inclusion of data due to yawning or related involuntary activity would further add to this situation. Possible misinterpretations are most likely to occur in situations where

the data acquisition system is highly automated and the results of individual trials are not routinely monitored for EMG artifacts. This situation is of paramount importance when working with hemiplegic patients who often appear fatigued and consequently have the potential for episodes of yawning. The potential for yawning's being a confounding variable in EMG bio feedback studies must be given serious consideration in both clinical and research studies. Although the exact physiological mechanism underlying yawning is yet to be established, it does appear to exert a strong facilitatory influence on the level of muscle activity generated by the hemiplegic patient.

\section{REFERENCES}

Basmajian, J. V. (1 985). Muscles alive.- Their functions revealed by electromyography (5th ed.). Baltimore: Williams \& Wilkins.

Bobath, B. (1978). Aduit hemiplegia: Evaluation and treatment (2nd ed.). London: Heinemann Medical Books.

Brunnstrom, S. (1956). Associated reactions of the upper extremity in adult patients with hemiplegia. Physical Therapy Review, 36(4), 225-236.

Cram, J. R., \& Garber, A. (1986). The relationship between narrow and wide bandwidth filter settings during an EMG scanning procedure. Biofeedback and Self-Regulation, 11, 105114.

Delwaide, P. J., \& Toulouse, P. (1 98 1). Facilitation of monosynaptic reflexes by 
voluntary contractions of muscles in remote parts of the body: Mechanisms involved in the Jendrassik manoeuvre. Brain, 104, 701-719.

Forrester, J. M. (1988). Is yawning a brainstem phenomenon? Lancet, March, 596.

Fugl-Meyer, A. R., Jaasko, L., Leyman, I., Olsson, S., \& Steglind, S. (1975). The poststroke hemiplegic patient: A method for evaluation of physical performance. Scandinavian Journal of Rehabilitation Medicine, 7, 13-33.

Hayes, K. C., \& Sullivan, S. J. (1976). Tonic neck reflex influence on tendon and Hoffmann reflexes in man. Electromyography and Clinical Neurophysiology, 16, 250-261.

Mulley, G. (1982). Associated reactions in the hemiplegic arm. Scandinavian Journal of Rehabilitation Medicine, 14, 177-120.

Sullivan, S. J., Mathieu, P. A., \& Goulet, C. (1989). The dilemma of EMG filter bandwidth selection for biofeedback studies -A deterministic investigation. Part 1: RMS analysis. Proceedingsof the2Oth AnnualMeetingof the Association for Applied Psychophysiology and Biofeedback (pp. 215-218). San Diego, California: Association for Applied Psychophysiology and Biofeedback.

Sullivan, S. J., Oman, R. E., Mathieu, P. A., Arsenault, A. B., \& Dutil, E. (1988). A template model, microcomputer based EMG biofeedback system for use with hemiplegic patients. Proceedings of the International Conférence of the Association for the Advancement of Rehabilitation Technology (pp. 430-431). Montreal, Quebec, Canada: RESNA.

Wimalaratna, H. S. K., \& Capiledo, R. (1988). Is yawning a brainstem phenomenon? Lancet, February, 300.

Wolf, S. L. (1978). Essential considerations in the use of EMG biofeedback. Physical Therapy, 58, 25-31. 
